\title{
THE EFFECT OF THE SOCIAL ORGANIZATION OF WORK ON THE VOLUNTARY TURNOVER RATE OF HOSPITAL NURSES IN THE UNITED STATES
}

\author{
Joan R. Bloom, 'Jeffrey A. Alexander ${ }^{2}$ and Beverly A. Nuchols ${ }^{1}$ \\ 'School of Public Health, University of California, Berkcley, CA 94720, U.S.A. and \\ ${ }^{2}$ School of Public Health, University of Michigan, Ann Arbor, MI 48109, U.S.A.
}

\begin{abstract}
In light of current concerns over nursing shortages and productivity, voluntary turnover among hospital nurses in the United States has assumed renewed importance as a managerial issue. This study examines the thesis that the social organization of work in hospitais is an important determinant of the voluntary turnover rate among registered nurses. This perspective differs from previous work in this area in that both turnover and its determinants are conceptualized at the organizational rather than individual level, thus opening the way for administrative intervention to reduce turnover. The conceptual model is tested using multiple regression techniques on a sample of 435 hospitals. Results suggest that organizational characteristics and environmental conditions are important contributors to turnover. Organizational characteristics are stronger predictors of turnover than are economic factors.
\end{abstract}

Key words-voluntary turnover, organizational turnover, nursing turnover, job dissatisfaction

\section{INTRODUCTION}

Over the last quarter century, a shortage of nurses to staff the hospitals in the United States has been a recurring concern [1]. In the 1960's shortages were attributed to women leaving the labor force during the childbearing years; federal programs were designed to increase the supply of nurses through incentives to training institutions to increase enrollment and facilitate reentry of nurses into the labor force. Interest of social scientists was directed toward understanding the reasons nurses were leaving the labor force and the problem was conceptualized from the perspective of the nurses' personal circumstances $[2,3]$.

During the late seventies, the shortages were attributed to the greater demand for nurse labor in certain specialties as a consequence of technological innovations. For example, innovations developed for use in the delivery of intensive care required more nurses. Increased career options for young women decreased the number of nurses entering the field. As the supply of new labor could not keep up with this increasing demand, hospitals began competing with each other to attract nursing staff [1]. Social scientists' analyses of turnover increasingly considered work related issues such as lack of professional autonomy and participation in hospital management, job stress and job dissatisfaction $[4,6]$.

High voluntary turnover rates at the beginning of the 1980's turned to involuntary turnover with the advent of prospective payment in 1983 [7]. Previously, hospitals were reimbursed by Medicare using a cost-based scheme in which patients (or insurers) were billed for the actual charges. With prospective reimbursement, hospitals were paid a fixed amount for hospital-based treatment for any one of 476 diagnoses. If costs per patient were less than this amount the hospital could keep the difference. If costs were in excess of this amount the additional monies came out of the operating budget of the hospital. This form of payment has produced pressures on hospitals to be more efficient. Hospital administrators have learned that survival under prospective payment requires cost containment. Nurses as the primary source of staffing are more efficient than the use of ancillary personnel. Yet, hospital costs associated with recruitment and hiring, personnel orientation and training have been shown to increase as a function of high turnover [3]. Excessive turnover has also been associated with diminished productivity and nursing effectiveness [6].

Recently, both the causes and solutions to voluntary turnover have been conceptualized and analyzed by social scientists as problems of the hospital organization rather than as a behavioral response of the individual nurse employee to personal factors or conditions of work $[8,9]$. The present study extends the organizational analysis of turnover by considering the predictors of nurse turnover in a sample of 435 hospitals. The social organization of work as an explanation of rates of voluntary turnover in hospitals is compared to an economic explanation of turnover.

\section{Background}

Research on turnover has been extensive in both the health $[5,6,8]$ and industrial sectors [11-13]. However, the estimated 1500 studies which have been reported in a number of reviews $[11,12]$ have resulted in relatively few noteworthy conclusions and in even fewer effective interventions to reduce turnover. Turnover has been examined largely at the individual 
level using attitudinal predictors such as job satisfaction or characteristics of the individual employee (e.g. age, education and marital status). Turnover among nurses specifically has been partially explained by personal factors such as marital status, short job tenure and first job incumbency $[8,14-18]$.

Employment conditions in hospitals have also been found to be related to voluntary turnover of individual nurses $[2,17,18]$. Turnover has been related to overtime work, rotating shifts, high workload, poor nurse leadership and poor relationships with physicians $[14,16-18]$. In Price and Mueller's [5] multivariate model of turnover, job specific variables such as communication, pay and promotional opportunities were related to turnover through their effects on job satisfaction and intent to stay. Personal factors such as education and kinship responsibilities affected turnover only through their relation to intent to stay. The results of a recent study using a multistage model to estimate turnover were consistent. Job satisfaction was weakly and indirectly related to turnover through its association with 'anticipated turnover'; personal factors did not have direct effects on turnover [8].

These studies demonstrate that characteristics of the work environment are predictors of job satisfaction. They suggest that turnover among individual nurses is mainly through 'intent to stay' or 'anticipated turnover'. However data for many of these studies have been collected in small samples of hospitals, raising the issue of generalizability of study findings to hospitals in different regions of the country, with different forms of ownership, and of different size.

\section{Turnover as an organizational attribute}

The analysis of voluntary turnover as an organizational attribute has a number of advantages. Shifting the explanation of turnover from individual to organizational attributes opens the way for administrative intervention through changes in practice related factors, organization design, and management policies $[18,19]$. At the aggregate level, turnover is also a measure of organizational performance and is a reflection of its general health and effectiveness [20-22]. It is one aspect of human resources management that is commonly monitored for both intervention and for making personnel planning projections. Finally, the information necessary to study turnover at the organizational level of analysis does not require measurement of perceptions, thoughts, and feelings of people which may be less reliable [21].

This study explores the thesis that the social organization of work is a key determinant of aggregate rates of nursing turnover in United States hospitals. Social organization of work may be defined as the structural characteristics of a work setting that, by design or default, affect the conduct of work in an organization. As a general construct, the social organization of work builds on several distinct theoretical traditions including professionals in organizations, technology and task design, organizational demography and organizational culture [20, 21, 23-26].

\section{Professionalization of the work force}

Professionalization of the workforce is a structural attribute and encompasses two dimensions along which the nursing staff can be defined-skill level and educational level. Typically, the nursing staffs of hospitals are composed of three key groups-registered nurses, licensed vocational (practical) nurses, and nursing assistants. Registered nurses are trained under a variety of educational approaches ranging from applied nurse training with a minimum of liberal arts and biological science to advanced training in both social and biological sciences. The relative mix of nurses with these educational backgrounds affects the prevailing ideology of nursing service as well as the nurses' commitment to the organization and their alternative opportunities for employment [20-25].

Economic theories of human capital as explicated by Becker [27] argue that the workers who have received specific training (on-the-job, task-specific training) are a better long-term investment for the employing organization as they are more likely to stay with the organization. This, in part, results because their task-specific training restricts their ability to move easily to other employment situations. By contrast, workers who have received professional training and/or general education offer task fexibility for the employing organization, but also have greater alternative opportunities outside the organization. This, coupled with strong professional (versus institutional) identification serve to enhance professionals' mobility and thus increases their likelihood of leaving. Empirical support for this assertion comes from Parsons [28] who studied turnover in a number of industries, including hospitals. In hypothesis one, we predict that in hospitals where the composition of the staff favors registered nurses higher turnover rates would be found, and hospitals whose nursing staffs have higher proportions of nurses with advanced degrees will experience the greatest turnover.

Hypothesis 1. The greater the professionalization of the hospital work force, the higher the voluntary turnover among registered nurses.

\section{Working conditions}

Resource use is the focal issue that distinguishes the administrative and professional models of nursing care and centers on the perception of the amount of resources necessary to provide appropriate nursing care. When hospital administration is responsible for organizing the work of staff nurses, issues of cost and efficiency predominate. Because they are removed from the process of patient care, administrators are more likely to perceive nursing activity as routine 
and accordingly staff the hospital to minimize costs. Staffing patterns dictated by administration generally are characterized by high patient to nurse ratios and by rotation of nurses between shifts, both cost containment strategies [19, 28, 29].

In general, this administrative view of work organization is not shared by professionally oriented nurses [30]. For example, when staffing patterns require the nurse to supervise the care of large numbers of patients irrespective of the acuity of the patient group, nurses become overworked and frustrated because the types of care consistent with their professional beliefs cannot be provided [31]. Role conflict engendered by the incompatibility of nurses' professional values and hospital bureaucracy has been cited as an explanation for exiting [16, 32]. Nurses who discover that their professional values are incompatible with the working conditions in the hospital are particularly vulnerable to turnover. Required shift rotation and lower staff to bed ratios are indicative of restrictive working conditions inconsistent with what professional nurses consider to be good nursing practice.

Hypothesis 2. The more restrictive the working conditions in the hospital, the higher the voluntary turnover among registered nurses.

\section{Professional work patterns and incentives}

Organizational units staffed by professional members function more effectively when practice patterns and work arrangements involve a high degree of discretion in decision making as well as collegial contact and exchange during the work process [25, 33-35]. The interaction with other professionals in the organization acts as a mechanism for reinforcing and sustaining the professional identity of the worker [36].

A key dimension of the professional conception of hospital organization concerns the existence of professional practice patterns in the hospital. Nurse practice patterns (e.g. primary nursing organizations) describe how work is organized in a particular patient care unit, specifically with regard to who makes patient care decisions, how much time a single nurse spends in caring for a group of patients, and how nurses will interact with patients, unit staff members, and other hospital personnel. The most professional practice patterns give the nurse maximum discretion in planning and carrying out the nursing care for patients (total patient care). Total care provided by the same nurse throughout the patient's hospitalization is called primary care; total care provided geographically is called modular care. Bureaucratic control is found in other practice patterns (team and functional nursing) [31]. In such patterns, the care giving tasks for each patient are divided among several members of the nursing staff and can be controlled by nurse-members of the administrative hierarchy. Hospitals, whose nursing staffs have greater participation in patient care decision-making should also experience less voluntary turnover.

A second dimension of professional structure in the hospital setting relates to opportunities for professional advancement. Following Thompson [24], hospital nursing has generally been defined as both an early and low ceiling occupation, one in which the gradations between entry level and top jobs are few. Further, the climb to the top position (ceiling) often arises before the individual's social needs for economic achievement have been satisfied. Until recently, professional career ladders were rarely found in hospital settings. In general, nurses had two choices for advancement, moving outside of the professional sphere into nursing administration or leaving the organization for alternative employment opportunities (e.g. community health nursing). Many hospitals have developed specialist roles for nurses as a means of retaining committed individuals with clinical expertise $[37,38]$. These specialist roles have several steps for advancement and as such are an alternative 'clinical' career ladder. As a second type of professional structure in the hospital, the existence of clinical career ladders for staff nurses is, therefore, expected to be related to lower nursing turnover.

Hypothesis 3. The more professionally designed the nursing service of the hospital, the lower the voluntary turnover among registered nurses.

In addition to the opportunities for interaction at work, professionally socialized workers also look to opportunities outside the work setting for professional growth and stimulation. These extrinsic job benefits, such as going to professional meetings, provide opportunities for increasing skills and collegial interaction, and are reinforced by professional organizations which require continuing education for relicensure. Therefore, it is expected that the provision of 'professional' work incentives to nurses will reduce nursing turnover by providing support for professional development outside the organization.

Hypothesis 4. The more professional work incentives offered by the hospital, the lower the voluntary turnover among registered nurses.

\section{Organizational demography}

Gaps in time of entry into the organization and the distribution of length of service among cohorts of hospital nurses have been associated empirically with turnover. Variation in cohort size at time of employment is thought to effect turnover for the following reasons: advancement opportunities within the organization may fluctuate providing less career mobility for some cohorts; the burden for socializing new cohorts may fall unequally among older cohorts resulting in inter-group tension; each cohort's experiences on entering the organization may be quite different and may result in conflict between the cohorts and difficulty in integrating dissimilar persons into the work units. Dominance of the longer 


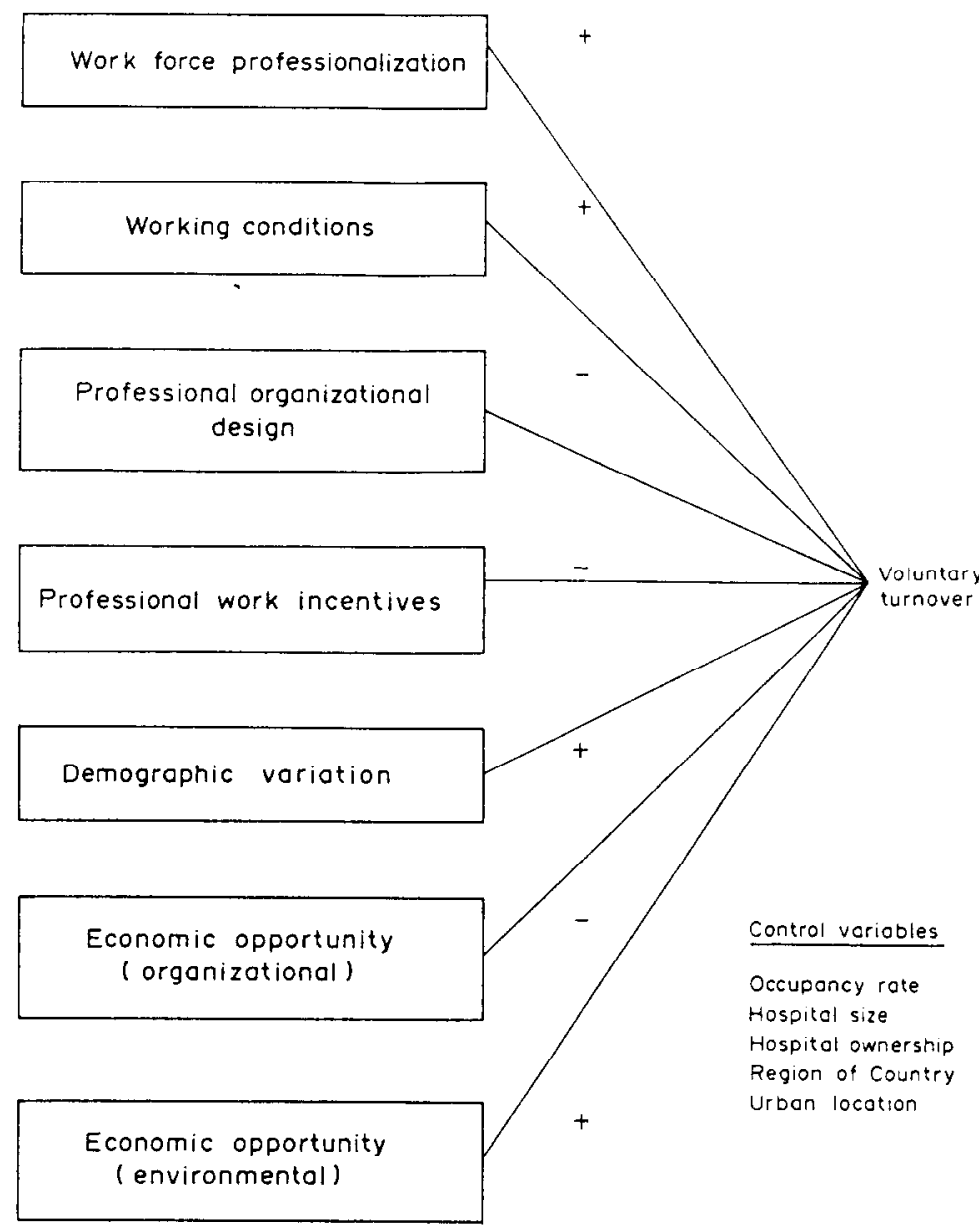

Fig. 1. Summary of predicted relationships between organizational characteristics and voluntary turnover of hospital nurses.

tenured cohort results in demographic stability. Organizations in which cohorts are more demographically variable due to date of hiring and length of tenure are found to experience greater turnover [21]. This finding is consistent with research on other organizations (e.g. universities) and holds up even when other factors which might predict turnover are controlled (local labor market, unemployment rate, wage rates, etc.) [39]. If one tenure cohort is larger and therefore, predominant in the organization, a less variable pattern exists. Following this line of reasoning it will be easier for new members to become integrated and they will be more likely to stay in the organization.

Hypothesis 5. The less variation in tenure cohort size within the nursing staff, the lower the voluntary turnover among registered nurses.

In sharp contrast to the social organization of work construct which emphasizes professional incentives and structures, models of turnover in the economics literature have emphasized extrinsic rewards such as wages and benefits and the opportunities for alternative employment as critical predictors of turnover [28, 40-43]. According to the economic thesis, turnover will be negatively related to the wages and benefits offered by the hospital (organizational economic opportunity) and positively related to the availability of local employment opportunities outside the hospital (environmental economic opportunity). This major alternative explanation will be operationalized to assess the independent effects of the social organization of work on turnover and the explanatory power of this construct relative to economically based explanations of turnover. Figure 1 summarizes the hypothesized relationships in the model.

\section{METHODS}

\section{Data Sources}

The primary source of data for this analysis was the Nurse Personnel Study conducted by the American Hospital Association [44] in 1981. The questionnaire elicited aggregate (hospital level) information on three aspects of the organization: (I) vacancies and turnover of both full-time and part-time nursing staff for inpatient services, outpatient services and specialty services (intensive care and coronary care), (2) work organization, including wages and fringe 
benefits, and recruitment practices, and (3) orientation programs for nurses. The questionnaire was addressed to the Chief Executive Officer of each hospital with the expectation that the Personnel Director's Office would assist in completing it. Because of the objective nature of the survey items, subjectivity bias was not a consideration in the data collection process. Telephone follow-up by A'HA staff was conducted to ascertain the reliability and accuracy of the data.

Data from two additional sources were merged to the Nursing Personnel Study data file. The 1981 AHA annual survey of hospitals and the Area Resource File. Data from the AHA annual survey of hospitals provided additional information on the hospitals general organizational structure. The Area Resource File provided county-level data on the external environment of the hospital.

\section{The Sample}

A $20 \%$ random sample (1223 hospitals) was drawn from a universe of approx 6110 hospitals throughout the country. The Nursing Personnel Survey was sent to hospitals in three waves with a telephone follow-up by AHA [44]. AHA Regional Directors were asked to encourage member hospitals to complete and return the questionnaire. These efforts yielded a sample of 732 hospitals (59.9\% response rate).

For the purposes of this analysis, a 'subsample' of AHA's sample was drawn using the following criteria: (1) hospitals were included if they reported four consecutive quarters of turnover data (1 January 1980 to 31 December 1980), (2) only hospitals reporting 10 or more full-time staff RNs were included to minimize magnification of personnel changes in small hospitals, and (3) only respondents providing complete turnover data for full-time registered nurses (RNs) were included to avoid contamination of turnover calculations through the inclusion of parttime registered nurses.

Missing data reduced the final usable sample to 435 hospitals. To insure that this 'subsample' was representative, a comparison was made to the original sample with regard to hospital size, region of the country, and ownership of the hospital. The subsample and the original sample were closely matched on two of these characteristics, size and ownership. However, in the subsample there was a slight overrepresentation of hospitals in the Northeast region of the country.

\section{Measurement}

\section{Dependent variable: voluntary turnover}

Two versions of the dependent variable, voluntary turnover, were developed (Table 1). In the first version, consistent with other studies, voluntary turnover rate was developed for descriptive purposes [5]. The numerator of this rate is based on the number of full-time registered nurses who voluntarily re- signed from their position from 1 January 1980 through 13 December 1980 (four calender year quarters). Individuals who were promoted, retired, fired, died or left due to disability were not included in this voluntary turnover calculation. The denominator consists of the mean number of registered nurses on staff for each quarter during the same period. Both turnover and staffing level data were reported by each hospital for each quarter; the overall measure was calculated by the researchers.

In the second version, developed for the multivariate model, the absolute number of full-time, registered nurses who voluntarily resigned their position was used as the dependent variable. The log of this voluntary turnover value was taken to normalize the distribution of the dependent variable in accordance with the assumptions of ordinary least squares regression [45]. The full-time registered nursing staff size was employed as an explicit control and is included as an independent variable in the regression model. Controlling for the effects of size in this fashion, eliminates problems of definitional dependency that occur through the use of ratio variables $[21,46]$.

\section{Independent variables: social organization of work}

Professionalization of the work force. Two measures were developed: (1) the skill level of the nursing staff which is measured as the ratio of registered nurses to total nursing staff and (2) the educational level of the nursing staff which is measured as the ratio of nurses with a baccalaureate, masters or doctoral degree to the total registered nursing (RN) staff. The first measure is designed to differentiate between technical education and on-the-job training and the second to assess the added effect of greater levels of education within the cohort of staff RNs [23].

Working conditions. Two dimensions of working conditions were measured. The $R N$ staffing ratio is defined as the proportion of full-time registered nurses on the nursing staff to the number of beds set up and staffed for use. Second, shiftwork is defined as the percentage of full-time registered nurses who rotate shifts.

Professional work patterns. The presence of professional nursing practice patterns in the hospital was defined as the proportion of hospital beds located in total nursing care, primary and modular nursing units. Standard definitions for each of these patient care patterns were provided in the survey [44]. Survey results indicate that $49.1 \%$ of the patient care was delivered by total patient care, $12.9 \%$ through primary care and $4.4 \%$ in modular care. The remainder of patient care was delivered through functional $(15.8 \%)$ and team nursing $(13.8 \%)$ or was not specified.

The second was the presence of a 'clinical' career ladder for the nursing staff in the hospital. Hospitals reporting $0-1$ clinical levels were coded as ' 0 ' while 
Table I. Means, standard deviations, and definitions of variables

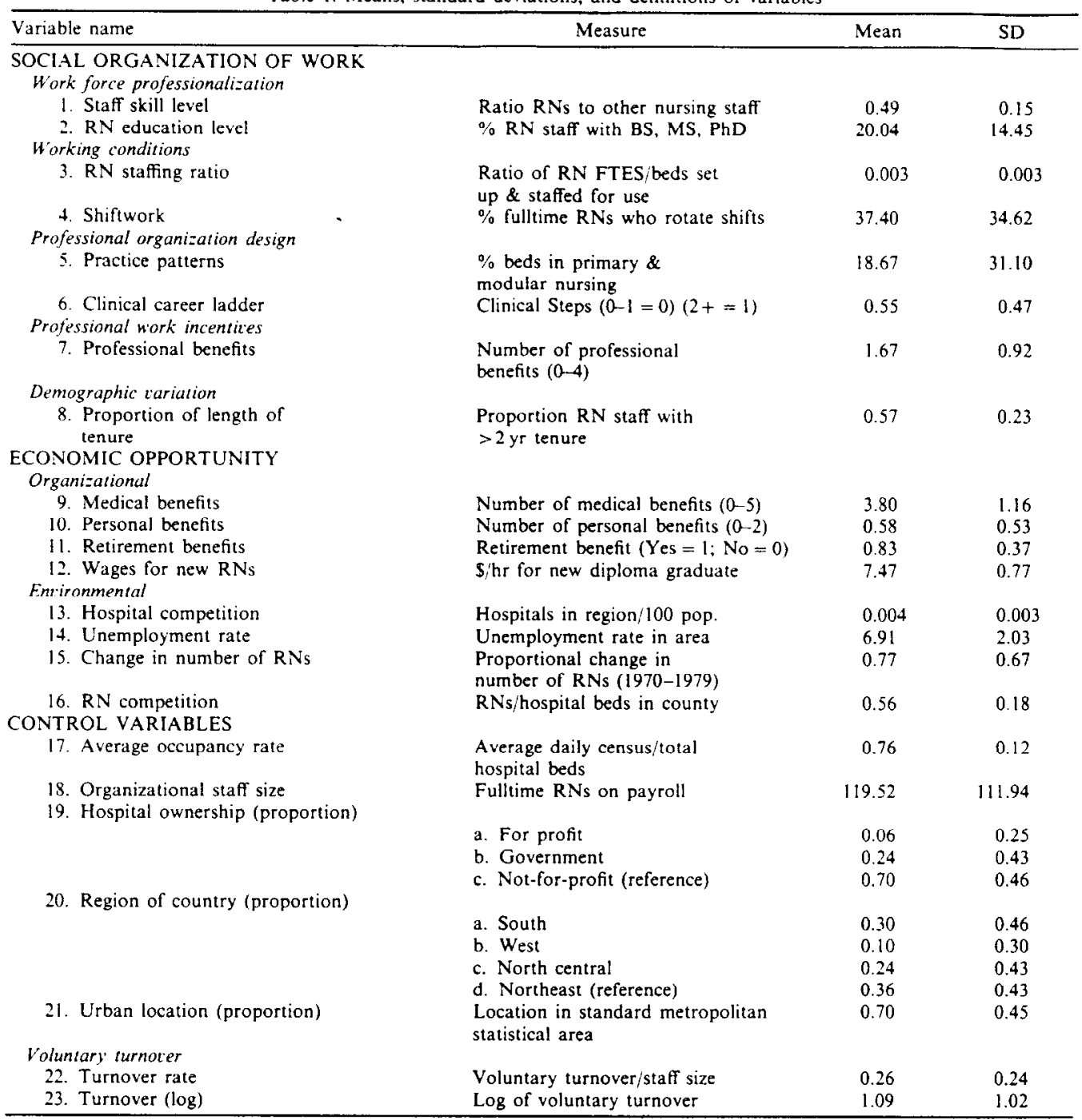

hospitals with two or more clinical levels were coded as ' 1 '.

Professional work incentives was measured as the sum of the following professional benefits provided through hospital sponsorship or subsidization: (1) continuing education tuition, (2) professional meeting fees, (3) professional membership fees and (4) clinical specialty certification.

Demographic variation. To assess the predominance of the longer temured nursing cohort within the hospital's demographic structure the proportion of the total nurses with a greater than a two year tenure was measured [47].

\section{Economic opportunity}

Economic opportunity at the organizational level and the environmental level was measured to rule it out as an alternative explanation for voluntary turnover. Four indicators of organizational level economic opportunity were included: (1) medical benefits (life insurance, basic health insurance, major medical/comprehensive insurance, dental insurance, and disability insurance); (2) personal henefits (maternity leave and child care); (3) retirement benefits; and (4) the starting wage for new diploma graduates. (The starting wage for new diploma graduates was chosen as a standardized measure of salary that would have applicability across hospitals.)

Four variables measuring economic opportunity at the environmental level are (1) hospital competition, defined as the ratio of hospitals in a county to its population; (2) the unemployment rate in the county; (3) the proportional change in the number of registered nurses in the county between 1970-1979; and (4) nurse competition, defined as the number of registered nurses per hospital bed per population in the county.

\section{Control variables}

Four hospital organization level variables were included: (1) the occupancy rate is the average daily census divided by total beds in the hospital. (2) Size 
Table 2. Turnover rate for size, region, location and ownership

\begin{tabular}{lrcc}
\hline & $N$ & Mean & SD \\
\hline All sample hospitals & 435 & 0.26 & 0.24 \\
Size & & & \\
$\quad$ Less than 99 beds & 80 & 0.23 & 0.21 \\
l00-300 beds & 209 & 0.25 & 0.16 \\
$\quad$ Greater than 300 beds & 146 & 0.31 & 0.34 \\
Region & & & \\
$\quad$ South & 131 & 0.31 & 0.25 \\
$\quad$ West & 44 & 0.38 & 0.50 \\
$\quad$ North Central & 105 & 0.23 & 0.13 \\
$\quad$ Northeast & 155 & 0.22 & 0.15 \\
Location & & & \\
$\quad$ Urban & 305 & 0.27 & 0.27 \\
$\quad$ Non-urban & 128 & 0.24 & 0.17 \\
Ownership & & & \\
$\quad$ For profit & 29 & 0.29 & 0.24 \\
$\quad$ Not-for-profit & 303 & 0.26 & 0.26 \\
$\quad$ Government & 103 & 0.26 & 0.20 \\
\hline
\end{tabular}

Two hospitals with missing data; $N=433$.

is defined as the average number of full-time RNs on the payroll from I January 1980 through 31 December 1980. (3) The ownership of the hospital is whether the hospital was investor-owned (for-profit), operated by state or local government, or operated as a voluntary, not-for-profit hospital (the reference group in the multivariate model). Two geographical measures of regional location were used. Region of the country was measured by a series of dummy variables corresponding to four geographical regions of the country-South, West, North-Central and Northeast (the reference group). Second, whether the hospital was located in an urban or rural location was measured. Hospitals located in an SMSA (Standard Metropolitan Statistical Area) were coded as ' 1 ', all others were considered non-urban and coded as ' 0 ' (Table 1).

\section{RESULTS}

The mean turnover rate for all sample hospitals was $0.26(\mathrm{SD}=0.24$ ) (Table 2$)$. Hospital size bore little relationship to turnover rates (the mean ranged from 0.23 to 0.31 ), although hospitals with more than 300 beds had a higher turnover rate than other size categories. Regional location, however, was strongly associated with turnover. Hospitals in the Northeast had the lowest turnover rates $(\bar{X}=0.22)$ while those in the West had the highest turnover rates $(\bar{X}=0.38)$. Whether the hospital was located in an urban or non urban area had little effect on turnover. Government $(\bar{X}=0.26)$ and not-for-profit hospitals had the lowest turnover rates $(\bar{X}=0.25)$ while for profit hospitals

Table 3. The effect of social organizations of work and economic opportunity on nursing turnover $(N=435)$

\begin{tabular}{|c|c|c|c|c|}
\hline Variable & $\boldsymbol{B}$ & SE & Beta & $T$-value \\
\hline \multicolumn{5}{|l|}{$\begin{array}{l}\text { SOCIAL ORGANIZATION OF WORK } \\
\text { Work force professionalization }\end{array}$} \\
\hline 1. Staff skill level & 1.68 & 0.35 & 0.25 & $4.73^{* * *}$ \\
\hline 2. RN educational level & 0.003 & 0.003 & 0.05 & 1.08 \\
\hline \multicolumn{5}{|l|}{ Working conditions } \\
\hline 3. RN staffing ratio & -117.16 & 19.59 & -0.29 & $-5.98 * * *$ \\
\hline 4. Shiftwork & -0.0003 & 0.001 & 0.01 & 0.227 \\
\hline \multicolumn{5}{|l|}{ Professional organization design } \\
\hline 5. Practice patterns & -0.002 & 0.001 & -0.07 & $-1.69 \#$ \\
\hline 6. Clinical career ladder & 0.08 & 0.09 & 0.04 & 0.89 \\
\hline \multicolumn{5}{|l|}{ Professional work incentives } \\
\hline 7. Professional benefits & 0.095 & 0.05 & 0.09 & $1.97^{*}$ \\
\hline \multicolumn{5}{|l|}{ Demographic variation } \\
\hline 8. Proportion of length of tenure & -0.67 & 0.19 & -0.15 & $-3.56^{* *}$ \\
\hline \multicolumn{5}{|l|}{ ECONOMIC OPPORTUNITY } \\
\hline \multicolumn{5}{|l|}{ Organizational } \\
\hline 9. Medical benefits & -0.06 & 0.04 & -0.07 & -1.48 \\
\hline 10. Personal benefits & 0.04 & 0.08 & 0.02 & 0.46 \\
\hline 11. Retirement benefits & 0.23 & 0.13 & 0.08 & $1.78 \#$ \\
\hline 12. Wages for new RNs & 0.15 & 0.06 & 0.12 & $2.37^{*}$ \\
\hline \multicolumn{5}{|l|}{ Entironmental } \\
\hline 13. Hospital competition & -36.95 & 17.78 & -0.10 & $-2.08^{*}$ \\
\hline 14. Unemployment rate in area & -0.02 & 0.03 & -0.05 & -0.93 \\
\hline 15. Change in number of $\mathrm{RNs}$ & 0.03 & 0.07 & 0.02 & 0.45 \\
\hline 16. RN competition & 0.59 & 0.31 & 0.10 & $1.91 \#$ \\
\hline \multicolumn{5}{|l|}{ CONTROL VARIABLES } \\
\hline 17. Average occupancy rate & -0.18 & 0.42 & -0.02 & -0.44 \\
\hline 18. Organizational staff size & 0.002 & 0.0004 & 0.23 & $4.67^{* * *}$ \\
\hline \multicolumn{5}{|l|}{ 19. Hospital ownership } \\
\hline (a) For profit (FP) & -0.13 & 0.18 & -0.03 & -0.71 \\
\hline (b) Government & -0.16 & 0.11 & -0.07 & -1.5 \\
\hline \multicolumn{5}{|l|}{ 20. Region of country } \\
\hline (a) South & 0.28 & 0.15 & 0.12 & $1.9 \#$ \\
\hline (b) West & 0.24 & 0.17 & 0.07 & 1.4 \\
\hline (c) North Central & 0.10 & 0.13 & 0.04 & 0.77 \\
\hline 21. Urban location & -0.33 & 0.11 & -0.15 & $-2.88^{* *}$ \\
\hline Intercept & -0.39 & 0.63 & - & -0.62 \\
\hline
\end{tabular}

Adjusted $R^{2}=0.35 ; d f=24,410 ; F=10.52$

$* * * P<0.0001$

$* * P<0.01$

$* P<0.05$.

$\# P<0.1$. 
had the highest rates $(\bar{X}=0.29)$. Zero order correlations between turnover. turnover rate and other study variables are presented in the Appendix.

To assess the simultaneous effects of the independent variables, we utilized an ordinary least squares regression model; all variables were entered simultaneously. The log of turnover for the hospital was regressed on the independent variables described in Table 1. The total equation is significant $(F=10.52$; df $24.410 ; P=0.001$ ), and explains $35 \%$ of the variance (see Table 3 ).

According to Hypothesis One, we expected to find that hospitals with more professionalized nursing staffs would have higher voluntary turnover than hospitals whose staffs were less professionalized. As predicted, the ratio of registered nurses to total nursing staff was significantly related to turnover (beta $=0.25 ; P<0.0001$ ). The other measure, the ratio of nurses with a BS, MS, or doctoral degree to total RN staff, had no independent effect on turnover.

Consistent with Hypothesis 2 the proportion of registered nurses on the nursing staff as a ratio to beds set up was negatively related to turnover (beta $=-0.29 ; P=0.0001$ ), but the percentage of full-time RN staff on rotation shifts was not associated with nursing turnover.

Hypothesis 3 and 4 consider the relationship of professional work design and professional incentives to nursing turnover. It was predicted that hospitals designed for professional employees, and those that provided more professional incentives would experience less turnover. The first indicator of the professionally designed organization was mode of patient care delivery. Controlling for the other variables in the model, the use of total, primary, and modular nursing organization for patient care had a significant but marginal effect on turnover (beta $=-0.07$; $P=0.09$ ). The existence of a career ladder was unrelated to turnover. However, the provision of professional benefits is related to higher rather than lower turnover in the hospital (beta $=0.09$, $P<0.05$ ), indicating lack of support for Hypothesis 4. In Hypothesis 5 we predicted that less variation in tenure cohorts within the hospital increases the stability of the workforce. Consistent with this hypothesis is the finding that hospitals with a larger proportion of longer tenured nurses have lower turnover (beta $=-0.15, P=0.0004$ ).

Economic opportunity has inconsistent effects on turnover. Consistent with the findings of Spencer [48] the hospital wage structure is related to turnover counter intuitively, i.e. hospitals with higher wages for beginning nurses are likely to have greater rather than lesser turnover (beta $=0.12 ; P=0.02$ ). Also, inconsistent with the economic opportunity explanation, hospitals in areas with greater competition have lower turnover. Also hospitals in the areas in which there are greater supply of nurses (to beds per county population) have higher turnover.

Two of the three control variables exhibited signifi- cant associations with turnover in the multivariate model. Consistent with expectations that hospitals with larger staffs experience greater turnover, organizations size was a powerful predictor in the model (beta $=0.23 ; P=0.0001$ ). Regional location of the hospital was also a significant determinant of nursing turnover. Hospitals in the South (beta $=0.12$; $P=0.06)$ experienced greater nursing turnover than those in the Northeast reference region. Ownership/control of the hospital, however, was not significantly related to turnover when other variables in the model were controlled.

As a final step, a commonality analysis was performed to identify the independent contributions of work organization and economic opportunity variables in explaining nursing turnover. The strategy of commonality analysis is based on separating the explained variance in turnover into portions unique to the social organization of work, economic opportunity, and the set of control variables. The unique contribution of the social organization and work set is the variance attributable to it when it is entered last into the regression model. It is represented as the squared semi-partial correlation between work organization and turnover after parceling out the effects of the alternative explanations [49]. The analysis addresses commonalities of variable sets rather than the individual measurement items. In this fashion, problems of interpreting higher order commonalities are avoided.

The $R^{2}$ difference between the combined regression model (control variables and social organization of work variables) and the regression containing only the control variables was $0.13(0.33-0.20)$. When the economic opportunity variables were substituted for the social organization of work set, this procedure resulted in an $R^{2}$ difference of only $0.03(0.23-0.20)$.

Based on a standard $F$ test, the independent contribution of the work organization variables for the model was significant at $F=9.85(P<0.001)$, while the contribution of the economic opportunity set was not significant $(F=1.36)$. As a theoretical construct, the social organization of work exercises a direct and independent effect on aggregate turnover among hospital nurses. The effect appears to be more salient for turnover than does economic opportunity.

\section{DISCUSSION AND CONCLUSIONS}

\section{Social organization of work}

Hospitals in which the nursing staff is composed of a higher ratio of registered nurses to other nursing personnel have greater turnover. This finding is consistent with earlier studies by both sociologists and economists $[5,28]$. It suggests the importance of the organizational culture in affecting behavior [50]. When nurses work in settings where there is a strong professional culture, their sense of their potential is reinforced, and alternative opportunities available to them are introduced. One might expect that turnover 
in this situation would be to another position rather than turnover due to family or other personal factors. Without individual level data, however, this line of inquiry cannot be pursued.

Second, turnover can also be explained by restrictive working conditions. Hospitals experiencing higher turnover were ones where the staffing ratio (ratio of the proportion of registered nurses to beds) was lower. The finding that staffing ratios are related to turnover is consistent with Prescott's study [19] who found that staffing ratios for both the day and night shift and nurses' perceptions of working conditions was related to turnover but that having nurse's rotate between shifts was not.

It is not surprising that measures of staffing are so important in the model. Staffing patterns, measured by staffing ratios, are influenced by the power relations between administration and nursing service. When the balance of power is in the favor of administration, lower staffing ratios, consistent with the view of the nurse as a non-professional worker, prevail. When nursing service is relatively more powerful, definitions of workload are based on professional considerations including the acuity of the patients being cared for, and professional standards of patient care delivery [31] which may increase the ratio of registered nurses to patients.

By focusing on the organizational context of the nurses' work, the effect of policies set by administrators for work within the organization can be seen. Our findings indicate that nurse staffing ratios as a measure of working conditions is an important determinant of turnover. Measures of working conditions reflect policies designed, perhaps, with short term cost savings in mind (such as increased productivity). The cost to the organization of voluntary turnover may not be considered.

Third, turnover was only partially affected by professional work patterns. It is somewhat surprising that a professionally oriented policy such as the existence of a 'clinical' career ladder had almost no effect on nursing turnover. Consistent with Prescott's findings [19] providing primary nursing care, which gives greater clinical decision making to the nursing staff, was only marginally related to lower levels of turnover (beta $=-0.07 P<0.09$ ). It may be that the former policy is less important because it does not effect the day to day work life of the nurse. Having responsibility for a group of patients and input into clinical decision making are daily experiences. An alternative interpretation of these findings is methodological. In most hospitals where clinical ladders existed, they were only two steps [38]. Differentiation between steps, both in terms of salaries and responsibilities, may not be sufficiently great to demonstrate a relationship with turnover $[39,40]$.

Contrary to our hypothesis, professional incentives increased voluntary turnover rather than lowered it. Coupled with the findings from Hypothesis 1, these data support the proposition that the existence of an organization with a professional culture promotes the development of a group of nurses who are professionally opportunistic. Of course, another interpretation is that hospitals with a professional culture may attract nurses who are already highly mobile. Where they are employed has little influence on their decisions to leave their nursing position. Prospective analyses are required to definitively explain these findings.

Finally, how well the hospital integrates new members into the social milieu of the organization is importantly related to turnover. Once a new member is recruited, organizational factors such as its demographic composition will affect whether the individual will stay or leave. Hospitals in which a majority of their nurses have been employed more than 2 years were found to have lower turnover than those dominated by younger members. This finding becomes even stronger when the larger and longer tenure group has been employed more than 5 years. This finding is consistent with the general argument that turnover will increase to the extent that work units are heterogeneous or dissimilar in terms of time of entry into the organization and in terms of distribution of length-of-service (tenure) among cohorts [21]. McCain and his colleagues [39] and Prescott [19] also found that the organization was more stable when there was less variability in tenure cohort size. In contrast to Hypothesis 1, where we found that a professionalized workforce may increase turnover, a predominant cohort with longer tenure may stabilize and reduce exits from the organization $[19,50,51]$.

\section{Economic opportunity: an alternative explanation}

Economic factors have independent effects on turnover but these effects, overall, do not make a statistically significant contribution to our model. Policies involving benefit structure seem to have little impact on turnover; the existence of a retirement benefit is the only exception and, like professional benefits is marginally related to higher turnover. This finding is consistent with the views of McCloskey [16] who separates factors which draw nurses to the job, and those that are important factors for exiting the organization. She found that salary and working conditions were important for taking a job while professional self-actualization was an important cause of turnover. Our results are inconsistent with economic theories of human capital which predict that higher starting wages of nurses and the existence of retirement benefits would be related to a lower quit rate [28]. One explanation is that hospitals already experiencing high turnover increase wages and benefits to attract nurses. The cross-sectional data does not allow us to establish a clear causal ordering between wages and benefits and turnover.

Environmental factors related to economic opportunity are also important in understanding the turnover phenomenon. Models of turnover in the 
economics literature have emphasized the importance of alternative employment options, typically indexed by the unemployment rate $[52,53]$ as well as competition between hospitals for staff [54]. Two of the measures of alternative employment opportunities were important. Contrary to March and Simon [52] and Halbur [55] we did not find the expected relationships between voluntary turnover and unemployment rate. Hospital competition (the number of hospitals per 100 population in the county) was a significant predictor of turnover. Contrary to expectations, hospitals in counties with higher ratios of hospitals to population experienced lower turnover. Examination of these data suggest it is the population density of the county (the denominator of the ratio) rather than the number of hospitals in the area (the numerator) that accounts for this finding. The decomposition of this ratio, suggests that the turnover rate is related to alternative employment opportunities provided in a higher population area, rather than to hospitals competing with each other for employees. In fact, discussions with hospital administrators support this explanation as they indicate that hospitals in close proximity differentiate services and their organizational cultures. Thus, nurses from other hospitals are not viewed as having a similar organizational culture and therefore, are of less interest to their hospital. Also inconsistent with the literature is the finding that increase in the supply of nurses, and presumably fewer alternative employment opportunities, is related to higher turnover.

\section{Other explanations: control variables}

Several of the control variables were also independently related to turnover. Larger hospitals experienced more turnover. Characteristics of hospital location were also important. Hospitals in counties with more hospitals experienced lower turnover, while those in nonurban locations and in the South experienced greater turnover. Since the sample was over-represented by hospitals in the Northeast region, generalization of the findings to all hospitals is tenuous.

It is not unexpected that registered nurse staff size would have such a profound effect on voluntary turnover as hospitals with larger staffs provide more opportunity for turnover. In this analysis, staff size was incorporated as an explicit control variable to avoid the problems of definitional dependency that occur by the use of ratio measures $[46,56]$. However, by including size as a control variable, the amount of variance explained may be artificially inflated.

A potential limitation of this study is the age of the data set. The data reflect salary, economic features and organization structures prevalent a decade ago and may not reflect contemporary circumstances. Recent reports, however, do not appear to support this concern. For example, the issue of wage compression and pay differentials as a function of experience and organization tenure are still major concerns
$[38,57,58]$ for nurses. In any case, since we are using the data to assess theoretical relationships rather than assess current situations, the age of the data is less problematic. We do not, for example, consider how actual salaries predict turnover as an economist might but instead look at differences between beginning salaries to see if relatively higher salaries decrease turnover. Even if the salaries are higher than they were when these data were collected, the assumption can be safely made that disparities would continue to exist and would not be relatively greater.

Whether or not to stay in a job or leave is an individual level decision, however, organizational factors play a major role. This analysis suggests that the organizational level of analysis is a fruitful way of conceptualizing turnover. From this perspective one can see how policies which relate to working conditions come to affect the organization's turnover rate. Administrators set policies for organizational staffing. These policies may be viewed by management as improving organizational efficiency by increasing the staff to patient ratio. However, these data suggest that such policies can have negative effects as well.

The findings from this study are important for policy making of hospital administration. Both norms of professional practice and patienl acuity should be explicit in developing policies for staffing and for delivery of patient care. Finally, administration should encourage those involved in orientation and inservice training to facilitate the integration of new cohorts of nurses.

In conclusion, this study explores an organizational analysis of turnover, and demonstrates the utility of conceptualizing the problem from this perspective. One direction for future research is to expand the model to include other organizational and environmental variables not captured in our model. One limitation of the current study is the use of cross sectional data. This limitation precludes establishing a relationship between wages and benefits and turnover, whether entry and exit factors differ or whether the organization's culture promotes mobility or it attracts a mobile group of nurses. Therefore, a second direction for future research is to collect longitudinal data to establish the causal ordering of variables. A third direction for research is to consider the costs to the institution of turnover. Finally, it is important to consider whether the problems faced in the United States have relevance to those faced in other countries by undertaking cross national comparisons of turnover of hospital nurses.

Acknowledgements - Support for this study came from the Committee on Research and the Institute of Industrial Relations, University of California, Berkeley. We thank the AHA for use of its data. We also thank Joan Chamberlain for her assistance in manuscript preparation. Earlier versions of this paper were presented at the American Sociological Association, New York City and at the Association of University Programs in Health Administration, Washington, D.C. 


\section{REFERENCES}

1. Aiken L. $\mathrm{H}$ and Mullinix C. F. The nurse shortage: myth or reality? N. Engl. J. Med. 13, 614-646, 1987.

2. Diamond L. and Fox D. Turnover among hospital staff nurses. Nursing Outlook 6, 388, 1958.

3. Knopf L. RNs one and five years after graduation: A report of the nurse career pattern study. Nat. League Nursing, 1975.

4. Weisman C., Alexander C. and Chase G. Job satisfaction and turnover among hospital nurses: $A$ longitudinal study. Hlth Services Res. 15,(4), 8-16, 1987.

5. Price J. and Mueller C. W. Professional Turnover: The Case of Nurses. SP Medical and Scientific Books, New York, 1981

6. Price J. and Mueller C. W. Absenteeism and Turnover of Hospital Employees. JAI Press, Greenwich, 1986.

7. Feder J., Hadley J. and Zuckerman S. How did Medicare's prospective payment system affect hospitals? N. Engl. J. Med. 317,(14), 867-873, 1987.

8. Hinshaw A. S., Smeltzer C. and Atwood J. Innovative retention strategies for nursing staff. $J$. Nursing $A d m i n$. $17,(6), 8-16,1987$

9. Alexander J. A. The effects of patient care unit organization on nursing turnover. Hlth Care Management Rev. 13, 61-72, 1988 .

10. Mobley W., Griffith H. H., Hand H. $H$. and Meglino B. M. Review and conceptual analysis of the employee turnover process. Psychol. Bull. 86, 493-522, 1979

11. Muchinsky P, and Tuttle M. Employee turnover: Empirical methodological assessment. J. Vocat. Behav. 14, 43-77, 1979

12. Bluedorn A. The theories of turnover: Causes, effects and meaning. In Research in Sociology of Organization (Edited by Bacharach S. B.), pp. 75-128. JAI Press, Greenwich, CT, 1982

13. O'Reilly C. and Caldwell D. The commitment and job tenure of new employees: Some evidence of postdecisional justification. Admin. Sci. Q. 26, 597-616, 1981.

14. Sloan F. The geographic distribution of nurses and public policy. In DHEW Publication (HRW) Health Manpower References. U.S. Government Printing Office, Washington, DC, 1975.

15. Kramer M. Nurse role deprivation: A symptom of needed change. Soc. Sci. Med. 2, 461-475, 1968.

16. McCloskey J. Influence of rewards and incentives on staff nurse turnover rate. Nursing Res. 23, 239-247, 1974.

17. Brief A. Turnover among hospital nurses: A suggested model. J. Nursing Administration 6, 55-58, 1976.

18. Everly G. and Falcione R. Perceived dimensions of job satisfaction for stafi registered nurses. Nursing Res. 25 , 346, 1976

19. Prescott P. A. Vacancy, stability and turnover of registered nurses in hospitals. Res. Nursing Hlth 9, 51-60, 1986.

20. Scott W. C., Dornbusch S., Evanswick C., Magnami L. and Sagatun I. In Technical Report \#47 Task Conceptions and Work Arrangements. Laboratory for Social Research, Stanford, CA, 1971.

21. Pfeffer J. and O'Reilly C. Hospital demography and turnover among nurses. Indust. Relations 26, 158-173, 1987.

22. Scott W, R. and Shortell S. Organizational performance: Managing for efficiency and effectiveness. In Health Care Management: A Text in Organization Theory and Behavior (Edited by Shortell S. M. and Kaluzney A. D.), pp. 418-461. Wiley, New York, 1988.

23. Georgopoulis B. and Mann F. The Community General Hospital. Macmillan, New York, 1962.
24. Thompson J. Organizations in Action. McGraw-Hill New York, 1967

25. Hall R. Professionalization and bureaucratization $A \mathrm{~m}$ Sociol. Rev. 33, 92-104, 1968.

26. Deal T. and Kennedy A. Corporate Culture. Addison Wesley, Reading, MA, 1982.

27. Becker $\mathbf{G}$. Investment in human capital: $A$ theoretical analysis. J. Political Econ. 76, 9-49, 1962.

28. Parsons D. Specific human capital: An application to quit rates and lay-off rates. $J$. Political Econ. 80 , $1120-1143,1972$.

29. Munson F. and Hedda S. An instrument for measuring satisfaction. Nursing Res. 23, 159,1974

30. Bloom J. R., O'Reilly C. and Parlette G. N. Changing images of professionalism: The case of public health nurses. Am. J. Publ. Hlth 69, 43-46, 1979.

31. Bloom J. R. and Alexander J. A. Team nursing: Professional coordination or bureaucratic control. J. Hlth Soc. Behav, 53, 84-95, 1982

32. Kramer $M$, and Baker $C$. The exodus: Can we prevent it? J. Nursing Admin. 1, 15-30, 1971.

33. Wandelt $\mathbf{M}$., Pierce $\mathbf{P}$. and Widdowson $\mathbf{R}$. Why nurses leave nursing and what can be done about it. $A m . J$ Nurs. 81,(1), 71-77, 1981 .

34. Engel G. Professional autonomy and bureaucratic organization. Admin. Sci. Q. 15, 12-20, 1970

35. Hall $R$. Some organizational considerations in the professional-organizational relationship. Admin. Sci. $Q$ 12,(3), 92-104, 1967.

36. Myrtle R. and Robertson J. Determinants of job satisfaction in nursing care units. J. Long-Term Care Admin. 7, 117-129, 1979.

37. Beyers H., Mullner R., Byre C. S. and Whitehead S. F Results of nursing personnel survey, Part 3: RN salary and fringe benefits. $J$. Nurs. Admin. 13,(6), 16-20, 1983.

38. Headline News: Study sees R.N. still gripped by pay compression. Am. J. Nurs. 90,(3), 93-112, 1990.

39. McCain B. E., O'Reilly C. and Pfeffer J. The effects of departmental demography on turnover: The case of a university. Acad. Management 26, 626-641, 1983.

40. Akerlof G. A., Rose A. K. and Yellen J. L. Job switching and job satisfaction in the U.S. labor market. Brookings Papers on Economic Activity, 19882 , 495-583, 1988

41. Weiss A. Determinants of quit behavior. J. Labor Econ 2,(3), 371-387, 1984.

42. Booton L. and Lane J. I. Hospital market structure and the return to nursing education. J. Hum. Resources 20,(1), 183-195, 1985

43. Holmlund B. and Lang $\mathbf{H}$. Quit behavior under imperfect information: Sending, moving, learning. Econ. Inquiry 23,(3), 383-393, 1985.

44. American Hospital Association. Nursing Personne Survey. Hospital Data Center, 840 N. Lake Shore Drive, Chicago, 1981

45. Chatterjee S. and Price B. Regression Analysis by Example. Wiley, New York, 1977.

46. Freeman J and Kronenfeld J. Problems of definitional dependency: The case of administrative intensity. Soc. Forces 52, 108-121, 1974.

47. Bloom J. R., Parlette G. N. and O'Reilly C. Collective bargaining by nurses: A comparative analysis of management and employee perceptions. Hlth Care Management Rev, 5, 25-33, 1980

48. Spencer D. Employee voice and employee retention Acad. Management J. 29, 488-502, 1986.

49. Kerlinger F. and Pehazur E. Multiple Regression in Behavioral Research. Holt, Rinehart and Winston, New York, 1973.

50. Camerer $\mathrm{C}$. and Vepsalainen $\mathrm{A}$. The economic efficiency of corporate culture. Strategic Management J. 9, 115-126, 1988. 
51. Hirschman A. O. Exit, Voice and Loyalty: Responses to Decline in Firms, Organizations and States. Harvard University Press, Cambridge. MA. 1970.

52. March J. and Simon H. Organizations. Wiley, New York, 1958.

53. Armknecht P. and Early J. Quits in manufacturing: A study of their cause. Monthly Labor Ret. 95, 31-37, 1972.

54. Robinson J. C. Hospital competition and hospital nursing. Nurs. Econ. 6,(3), 116-119, 1988.
55. Halbur B. Nursing Personnel in nursing homes: A structural approach to turnover. Organizations Occupations 10,(4), 381-411, 1983.

56. Teaborg J. and Lee T. A predictive study of organizational turnover rates. Ac. Management J. 27, 793-810, 1984.

57. Headline News: New salary wars promise solid gains this year. Am. J. Nurs. 88, 113, 117, 120-122, 1988.

58. Headline News: RN Vacancies Hit $12.66 \%$ in 1989. Am. J. Nurs. 90, 11, 1990.

\section{APPENDIX}

Inter Correlations Between Social Organization, Economic Opportunity, Control Variables and Two Measures of Turnover

\begin{tabular}{|c|c|c|}
\hline & Turnover & Turnover rate \\
\hline 1. Staff skill level & 0.30 & 0.06 \\
\hline 2. RN educational level & 0.22 & 0.20 \\
\hline 3. RN staffing ratio & -0.33 & -0.08 \\
\hline 4. Shiftwork & 0.12 & 0.05 \\
\hline 5. Practice pattern & 0.05 & 0.02 \\
\hline 6. Clinical career ladder & 0.02 & 0.03 \\
\hline 7. Professional benefits & 0.07 & 0.04 \\
\hline 8. Proportion of length of tenure & -0.21 & -0.28 \\
\hline 9. Medical benefits & 0.06 & -0.00 \\
\hline 10. Personal benefits & -0.01 & 0.04 \\
\hline 11. Retirement benefits & 0.11 & -0.00 \\
\hline 12. Wages for new RNs & 0.20 & 0.18 \\
\hline 13. Hospitals competition & -0.20 & 0.01 \\
\hline 14. Unemployment rate & -0.11 & -0.08 \\
\hline 15. Change in number of $\mathrm{RNs}$ & 0.02 & 0.08 \\
\hline 16. RN competition & 0.24 & 0.07 \\
\hline 17. Average occupancy rate & 0.17 & -0.09 \\
\hline 18. Organization's staff size & 0.44 & -0.05 \\
\hline \multicolumn{3}{|l|}{ 19. Hospital ownership } \\
\hline For profit (FP) & -0.03 & 0.03 \\
\hline Government & -0.15 & -0.00 \\
\hline Not-for-profit & 0.14 & -0.01 \\
\hline \multicolumn{3}{|l|}{ 20. Region of country } \\
\hline South & -0.02 & 0.12 \\
\hline West & $0.0 \mathrm{I}$ & 0.16 \\
\hline North central & 0.05 & -0.09 \\
\hline Northeast & -0.03 & -0.14 \\
\hline 21. Urban & 0.18 & 0.06 \\
\hline
\end{tabular}

$0.11, P=0.05$.

$0.16, P=0.01$

$0.19, P=0.00 \mathrm{I}$. 